\title{
Relationship between the degree of abdominal wall movement and the image quality of contrast-enhanced MRI: semi-quantitative study especially focused on the occurrence of transient severe motion artifact
}

\author{
Hiroshi Ikeno ${ }^{1}$ - Satoshi Kobayashi ${ }^{1,2}$ (D) Kazuto Kozaka ${ }^{1} \cdot$ Takahiro Ogi $^{1} \cdot$ Dai Inoue $^{1} \cdot$ Norihide Yoneda $^{1}$. \\ Kotaro Yoshida $^{1} \cdot$ Naoki Ohno $^{2} \cdot$ Toshifumi Gabata $^{1} \cdot$ Azusa Kitao $^{1}$
}

Received: 21 February 2019 / Accepted: 24 October 2019 / Published online: 5 November 2019

(c) Japan Radiological Society 2019

\begin{abstract}
Purpose To reveal the relationship between abdominal movement and artifact, and to reveal if the transient artifact in arterial phase is caused by transient abdominal movement (TAM) in contrast-enhanced (CE) MRI.

Materials and methods 325 CE-MRI series (206 with EOB and 119 with EGCM) were included. The abdominal movement was classified into three groups by respiratory bellows waveform (= bellows grade, BG $1-3)$, and MR image quality (= artifact score, AS) was graded 1-5 for the precontrast, arterial and portal venous phase, respectively. The relationship between the BG and AS was evaluated. The occurrence of transient artifact in arterial phase was compared to the degree of TAM. Results In the acquisitions with BG3, all images showed AS of $>2$, while no images had AS of $>4$ in the acquisitions with BG1. Numbers of transient artifact in the arterial phase with no-abdominal movement (NAM), mild-TAM, severe-TAM were 0 of 120, 4 of 27, 7 of 8 in EOB and 0 of 91, 1 of 4, 0 of 0 in EGCM, respectively.

Conclusion Image quality is highly correlated with abdominal movement. Moreover, artifact in arterial phase was not observed in NAM, which indicated abdominal movement is the direct cause of artifact.
\end{abstract}

Keywords Magnetic resonance imaging (MRI) $\cdot$ Gd-EOB-DTPA $\cdot$ Liver $\cdot$ Transient severe motion · Artifact (TSM) · Respiratory bellows

\section{Introduction}

Gd-EOB-DTPA (EOB) is a new gadolinium-based contrast agent, which can assess both the hepatic perfusion and function at the same examination. It is widely used for the detection and characterization of focal liver lesions [1-3].

However, there are several reports that the image deterioration of arterial phase of dynamic MRI is more often observed in EOB-enhanced MRI than in gadobenate

Satoshi Kobayashi

satoshik@staff.kanazawa-u.ac.jp

1 Department of Radiology, Kanazawa University Graduate School of Medical Sciences, 13-1 Takara-machi, Kanazawa 920-8641, Japan

2 Department of Quantum Medical Technology, Kanazawa University Graduate School of Medical Sciences, 13-1

Takara-machi, Kanazawa 920-8641, Japan dimeglumine-enhanced MRI [4-7]. This arterial phase image deterioration on dynamic contrast-enhanced MRI is called transient severe motion (TSM) artifact [4, 5].

Arterial phase image deterioration can be caused by either truncation artifact by rapid change of signal intensity in the arteries during arterial phase acquisition, or TSM. Most papers about TSM speculated that the cause of artifact was abdominal movement by breath-hold failure [8,9], but it has not been proved yet. We hypothesized that transient abdominal movement (TAM) during arterial phase image acquisition period might be observed in cases with positive TSM; furthermore, no abdominal movement cases during arterial phase cannot show TSM.

This study was conducted to reveal the direct relationship between abdominal movement and artifact, and to reveal if the transient artifact in arterial phase (so-called TSM) is caused by TAM, both in Gd-EOB-DTPA (EOB)- and 
other extracellular gadolinium contrast material (EGCM)enhanced MRI.

\section{Materials and methods}

This retrospective study was approved by our institutional review board and informed consent was waived.

In this study, we termed image deterioration observed after contrast injection as "transient artifact in arterial phase" to discriminate it from abdominal movement recorded with respiratory bellows.

\section{Data acquisition of EOB-enhanced MRI group (EOB group) and EGCM-enhanced MRI group (EGCM group)}

Total 245 patients underwent contrast-enhanced MR study with EOB (Primovist; Bayer HealthCare, Osaka, Japan) from February 2011 to February 2012. Thirty-nine patients were excluded because of lack of patient demographic data and/or repeated MR imaging. Finally, EOB group consisted of 206 patients (78 women and 128 men).

Total 127 patients underwent contrast-enhanced MR study with EGCM [Omniscan, Daiichi Sankyo, Tokyo, Japan $(n=18)$; Magnescope, Fuji Pharma, Tokyo, Japan $(n=11)$; Gadovist, Bayer HealthCare, Osaka, Japan $(n=54)$; or Prohance, Eisai, Tokyo, Japan $(n=36)]$ from November 2015 to June 2016. Eight patients were excluded because of lack of patient demographic data and/or loss of the respiratory bellows waveform data. Finally, 119 patients who underwent contrast-enhanced MR study with EGCM were included in this group (57 women and 62 men).

\section{Patient characteristics}

Patient characteristics and risk factors, including patient sex, age, body weight, body mass index (BMI), smoking exposure (pack-years), ascites, and pleural effusions, that could predispose to respiratory motion artifacts were recorded both in EOB group and in EGCM group.

\section{Image acquisition}

All the MR examinations were performed with a 3-T MR system (Signa HDx; GE Medical Systems, Milwaukee, WI, USA). Precontrast images were obtained in a transverse plane with a fat-suppressed three-dimensional (3D) T1-weighted gradient echo (GRE) sequence (LAVA-XV, $\mathrm{TR} / \mathrm{TE}=3.2-4.0 / 1.6 \mathrm{~ms}$, flip angle $12^{\circ}$, field of view $36 \times 36 \mathrm{~cm}$, matrix $320 \times 192$, interpolated to $512 \times 512$, slice thickness $4.2 \mathrm{~mm}$, overlap $2.1 \mathrm{~mm}$, sequential view ordering).
In EOB-enhanced MRI, all patients received a dose of $0.1 \mathrm{~mL} / \mathrm{kg}$ EOB intravenously at a speed of $1.0 \mathrm{~mL} / \mathrm{s}$ and the line was flushed at a speed of $1.0 \mathrm{~mL} / \mathrm{s}$ with $20 \mathrm{~mL}$ of saline. Immediately after the start of the EOB injection, dynamic studies with a fat-suppressed 3D T1-weighted GRE sequence were performed using the test injection method ( $0.5 \mathrm{~mL}$ of Primovist with $8 \mathrm{~mL}$ saline flush), and arterial phase timing was determined as the peak time of the abdominal aorta plus $10 \mathrm{~s}$ minus half of imaging time. According to this formula, arterial phase timing was approximately $25 \mathrm{~s}$ after the start of EOB injection. Portal venous and transition phase images were obtained at $60-90$ and $120-180 \mathrm{~s}$ after the start of EOB injection.

In EGCM-enhanced MRI, arterial phase was imaged at a fixed time of $25 \mathrm{~s}$ after the initiation of EGCM injection. Portal venous and equilibrium phase images were obtained at 60-70 and 130-140 s after EGCM injection. Among the previously listed EGCM, patients with Gadovist enhancement were administered a dose of $0.1 \mathrm{~mL} / \mathrm{kg}$ intravenously at a speed of $1.0 \mathrm{~mL} / \mathrm{s}$. The line was flushed at a speed of $2.0 \mathrm{~mL} / \mathrm{s}$ with $20 \mathrm{~mL}$ of saline. Patients with the other EGCM enhancement were administered a dose of $0.2 \mathrm{~mL} /$ $\mathrm{kg}$ intravenously at a speed of $2.0 \mathrm{~mL} / \mathrm{s}$. The line was flushed at a speed of $2.0 \mathrm{~mL} / \mathrm{s}$ with $20 \mathrm{~mL}$ of saline.

\section{Assessment of the abdominal movement with waveform of respiratory bellows}

Electrograms of respiratory bellows waveform during precontrast, arterial and portal venous phase image acquisition period stored as jpeg image file were used for the analysis of the degree of abdominal movement. We categorized the waveform of the respiratory bellows into the following three respiratory bellows grades: grade 1 (no movement), grade 2 (mild movement) and grade 3 (severe movement). Grade 1 shows no fluctuation of the baseline of electrogram during breath-hold period of the contrast-enhanced MR image acquisition, which represents good breath-hold and thus no abdominal movement (Fig. 1a). Grade 2, which represents respiratory bellows waveform of mild movement, shows minimal to moderate fluctuation of the baseline of electrogram during breath-hold period of the contrast-enhanced MR image acquisition. Its amplitude keeps less than onethird of that of observed under normal respiration process (Fig. 1b). Grade 3, which represents respiratory bellows waveform of severe movement, shows irregular, polymorphic and bigger amplitude of fluctuation of the baseline of electrogram during breath-hold period of the contrastenhanced MR image acquisition. Usually its amplitude of fluctuation is bigger than the normal respiratory state, which represents the occurrence of severe abdominal movement (Fig. 1c). In some cases, the baseline of electrograms of respiratory bellows waveform during breath-hold period 


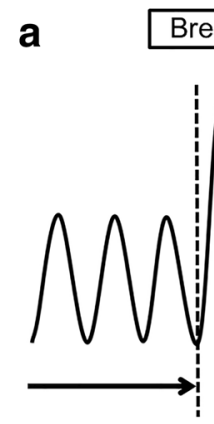

Free Breathing Period
Breath Hold

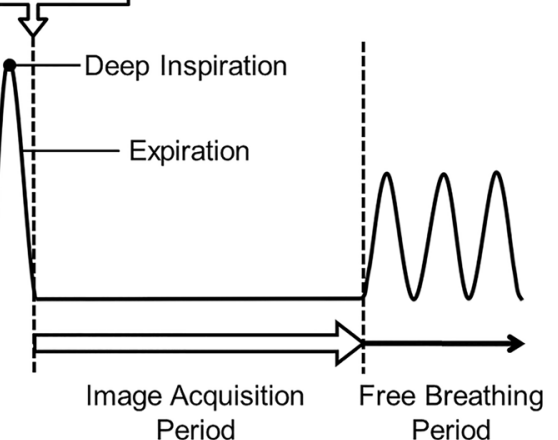

C

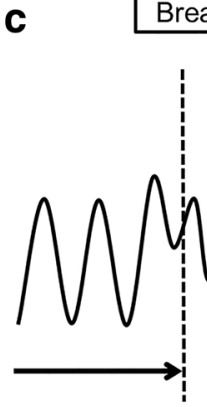

Free Breathing Period

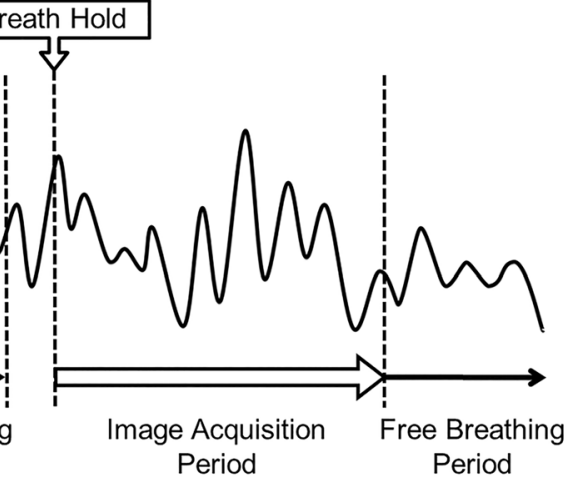

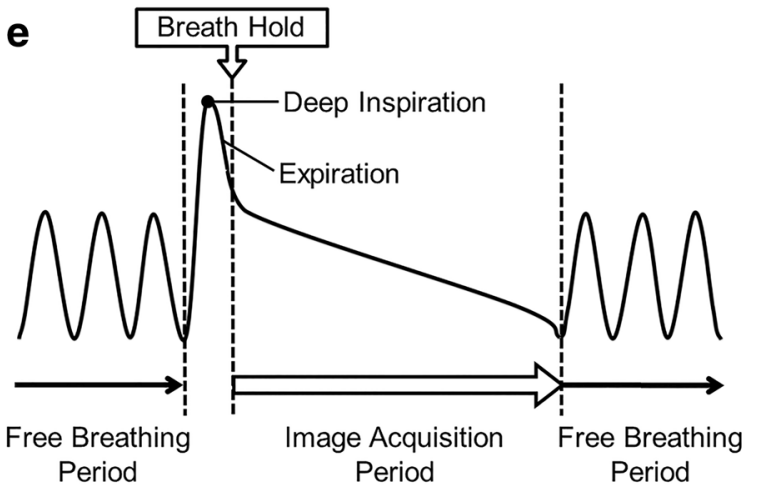

Fig. 1 Schematic diagrams of electrogram of respiratory bellows waveform patterns of each respiratory bellows grades. a Respiratory bellows grade 1, which represents no fluctuation of the baseline of electrogram during breath-hold period of the contrast-enhanced MR image acquisition. b Respiratory bellows grade 2, which represents respiratory bellows waveform of mild movement. Its amplitude keeps less than one-third of that of observed under normal respiration process. c Respiratory bellows grade 3, which represents respiratory bellows waveform of severe movement, shows irregular, polymorphic

may show not fluctuation but upward or downward curve (Fig. 1d, e). We consider these subtle waveform variations
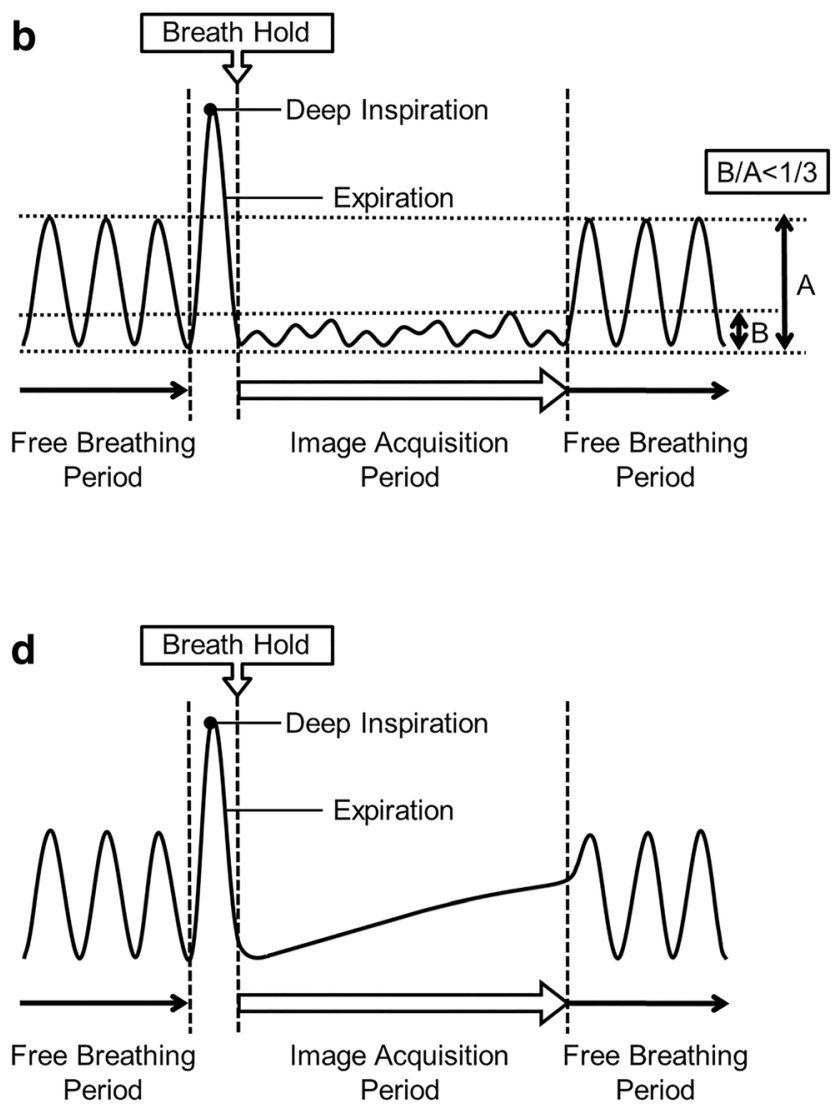

and bigger amplitude of fluctuation of the baseline of electrogram during breath-hold period of the contrast-enhanced MR image acquisition. Usually its amplitude of fluctuation is bigger than normal respiratory state, which represents the occurrence of severe abdominal movement. In some cases, baseline of electrograms of respiratory bellows waveform during breath-hold period may show not fluctuation but upward (d) or downward (e) curve. These subtle waveform variations represent minimal abdominal movement and include them in respiratory bellows grade 2

represent minimal abdominal movement and include them in respiratory bellows grade 2 group. 
After anonymized and randomized, total 975 electrogram images of respiratory bellows (in 206 EOB-group and 119 EGCM-group patients) were reviewed and categorized into the three bellows grades by 2 readers (K. K. and H. I.). In case of disagreement, the final decision was reached by consensus.

\section{Assessment of the MR image deterioration with artifact score}

Total 975 MR image series obtained in precontrast and dynamic postcontrast (arterial and portal venous) phases of 325 patients were anonymized, randomized, and reviewed by three radiologists (K. K., A. K, and T. O.), each with more than 12 years of experience in the interpretation of abdominal imaging. The reviewers assessed the image series in terms of the degree of the image deterioration presumably caused by abdominal movement. These readers were specifically instructed to ignore other sources of artifacts, particularly truncation artifact. Here, our definition of truncation artifact is as follows: (1) bright or dark lines parallel to the edge of the interface; (2) artifactual false widening of edges at these interfaces.

We defined the artifact score as follows [4]: score 1, no image deterioration (Fig. 2a); score 2, minimal respiratory motion-related artifacts with minimal image deterioration and no effect on diagnostic quality (Fig. 2b); score 3, moderate respiratory motion-related artifacts with some, but not severe image deterioration, which is acceptable for diagnostic use (Fig. 2c); score 4, severe respiratory motion-related artifacts with considerable image deterioration; however, images were still acceptable for diagnostic use (Fig. 2d); score 5, extensive respiratory motion artifacts with severe image deterioration and images were not acceptable for diagnostic use (Fig. 2e).

\section{Analysis of relationship between respiratory bellows grade and artifact score}

Relationship between the degree of the abdominal movement, which is represented by respiratory bellows grade, and the severity of the image deterioration which is represented by the average artifact score is analyzed in EOB group and EGCM group.

\section{Classification of abdominal movement pattern in contrast-enhanced MRI}

To assess the relationship between abdominal wall movement measured by respiratory bellows and the occurrence of transient artifact in arterial phase (so-called TSM), we classified abdominal movement patterns of pre-contrast, arterial phase and portal phase into the following categories (Table 1).

At first, we considered patients with grades 2 and 3 in precontrast image as having difficulty in breath-holding extraneous to the contrast material administration. Then we defined no abdominal movement (NAM) as respiratory bellows grade 1 in all phases.

Afterwards, we defined transient movement of the abdominal wall observed only during arterial phase as TAM, namely when the contrast-enhanced image series meets the following two criteria $(a, b)$ at the same time, we define the case as positive TAM: (a) respiratory bellows grade 2 or 3 in arterial phase and (b) respiratory bellows grade 1 in precontrast and portal venous phase. Then TAM cases are subdivided into the following two groups: arterial phase respiratory grade 2 group is defined as mild-TAM group and arterial phase respiratory grade 3 group is defined as severeTAM group.

Finally, we defined the cases which the contrastenhanced image series did not meet the previous criteria (for example, respiratory bellows grade 1 both in precontrast and arterial phase and respiratory bellows grade 2 or 3 in portal venous phase) as irregular abdominal movement (IAM) case.

\section{Relationship between the degree of TAM and the presence of transient artifact in arterial phase}

The frequency and the degree of each abdominal movement pattern in both EOB group and EGCM group were recorded respectively. To show the direct relation between the abdominal movement and image deterioration, relationship between the degree of TAM and the occurrence of the "transient artifact in arterial phase" were analyzed both in EOB group and in EGCM group. Moreover, average artifact scores on each phase of dynamic contrast-enhanced MRI in NAM, mild-TAM and severe-TAM cases are compared both in EOB group and EGCM group (Fig. 3).

Since IAM cases and "difficulty in breath-holding" cases are unsuitable for analysis of the "transient artifact in arterial phase", we excluded these cases in this analysis.

Artifact score of 4 or greater was considered as severe artifact. When the contrast-enhanced image series meets the following two criteria $(a, b)$ at the same time, we define the case as positive "transient artifact in arterial phase" case [6, 7]: (a) severe artifact in arterial phase (artifact score 4 or 5) and (b) artifact score 2 or less in precontrast and portal venous phase (Fig. 4). 

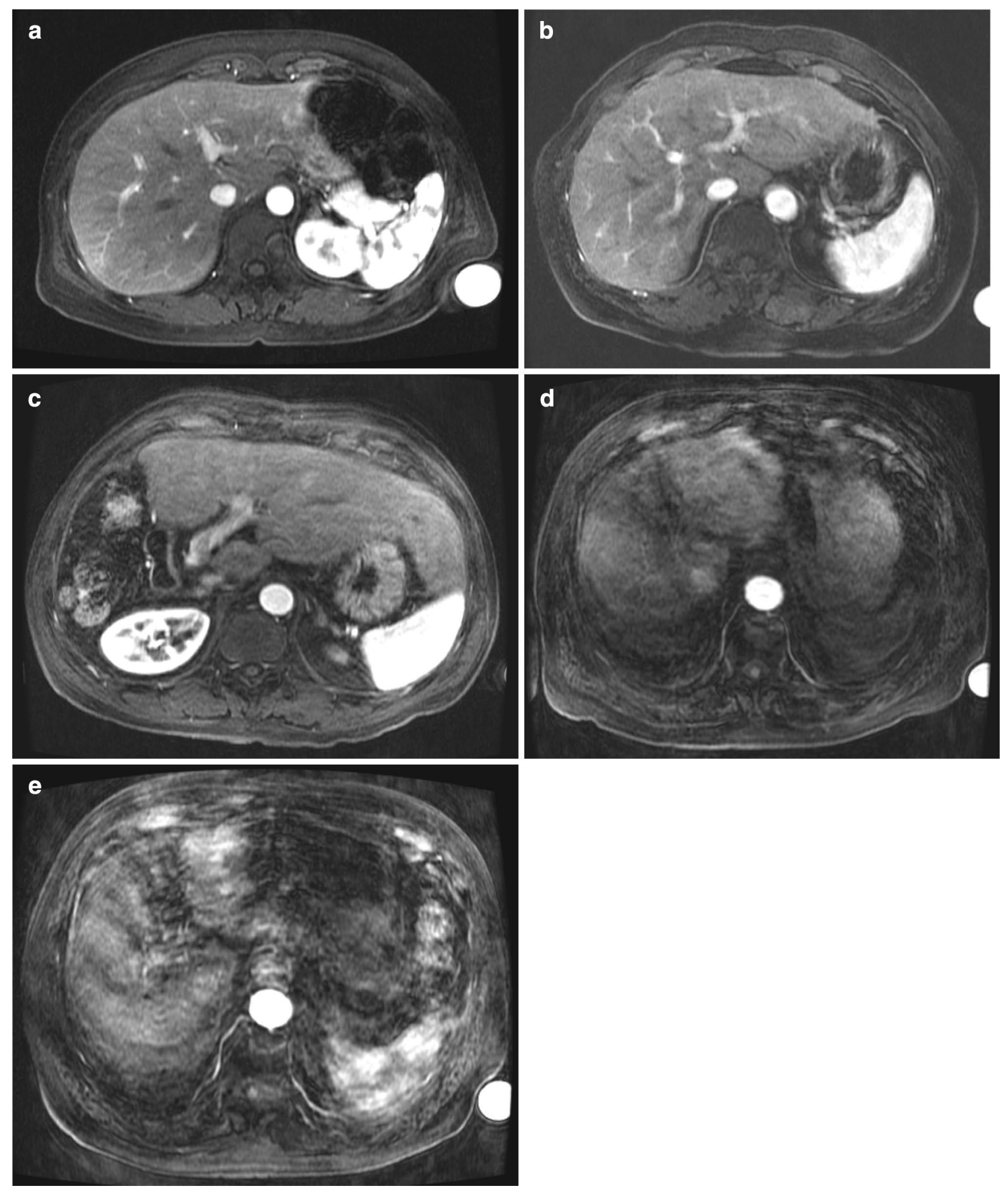

Fig. 2 Example of each artifact score images. a Score 1: no image deterioration. b Score 2: minimal respiratory motion-related artifacts with minimal image deterioration and no effect on diagnostic quality. c Score 3: moderate respiratory motion-related artifacts with some, but not severe image deterioration, which is acceptable for diagnostic use. d Score 4: severe respiratory motion-related artifacts with considerable image deterioration however images were still acceptable for diagnostic use. e Score 5: extensive respiratory motion artifacts with severe image deterioration and images were not acceptable for diagnostic use 
Table 1 Classification of abdominal movement pattern in contrastenhanced MRI

\begin{tabular}{llll}
\hline & \multicolumn{3}{l}{ Contrast-enhanced phase } \\
\cline { 2 - 4 } & Precontrast & AP & PVP \\
\hline $\begin{array}{l}\text { Abdominal movement pattern } \\
\text { Difficulty in breath-hold }\end{array}$ & G2 & Any & Any \\
No abdominal movement (NAM) & G1 & & \\
$\begin{array}{l}\text { Transient abdominal movement (TAM) } \\
\text { Mid TAM }\end{array}$ & G1 & G1 & G1 \\
$\quad$ Severe TAM & & G2 & G1 \\
$\begin{array}{l}\text { Irregular abdominal movement } \\
\text { (IAM) }\end{array}$ & Other combinations & & \\
\hline
\end{tabular}

G1 bellows grade 1, G2 bellows grade 2, G3 bellows grade 3, any any bellows grade, $A P$ arterial phase, $P V P$ portal venous phase
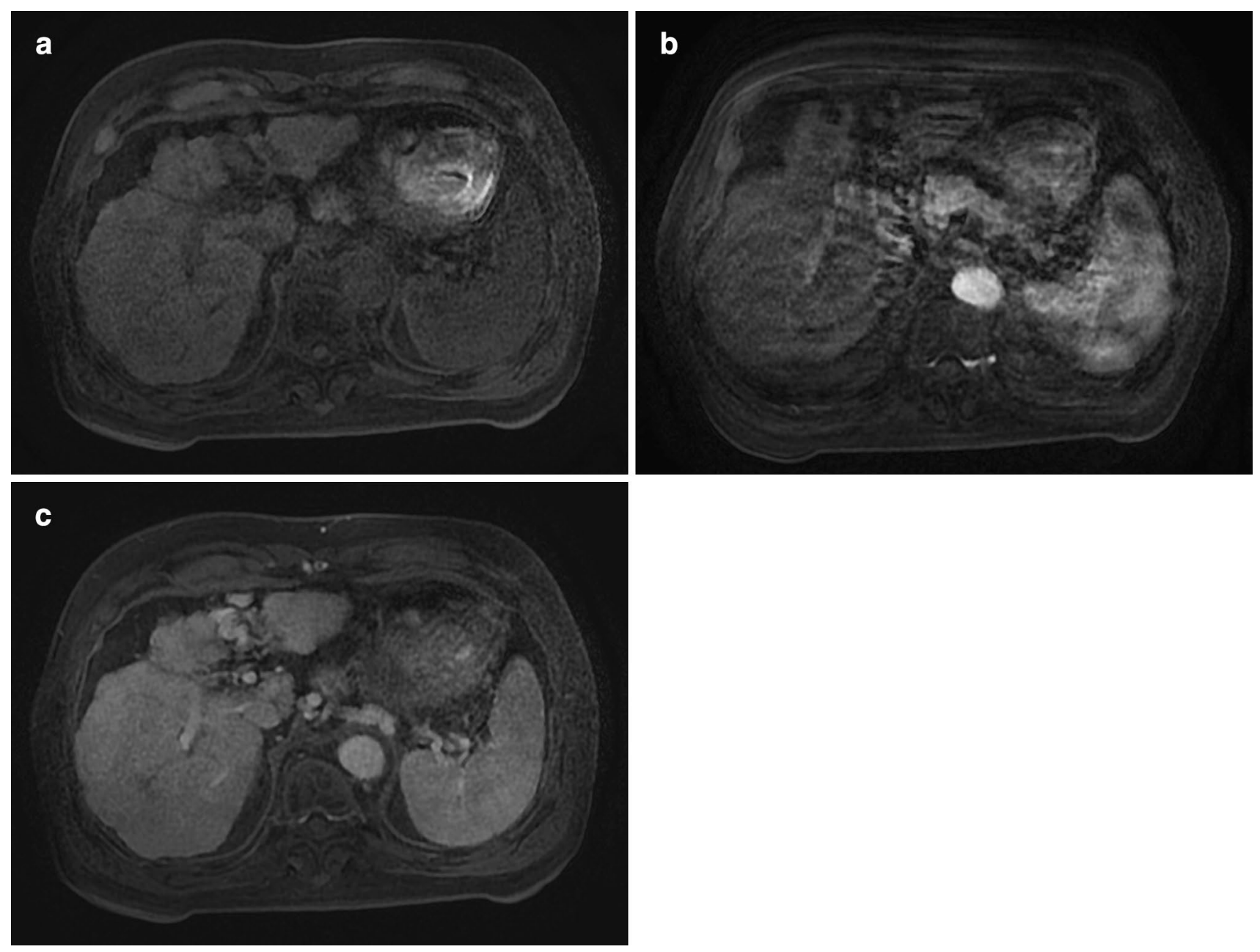

Fig. 3 Example of positive "transient artifact in arterial phase" case (images are obtained from the same patient). a Precontrast image. b Arterial phase image. c, Portal venous phase image. Both precontrast image (a) and portal venous phase image (c) showed artifact score

\section{Statistical analysis}

The statistical tests were performed with the commercially available software (SPSS version23; IBM, Armonk, NY and Prism version 6; GraphPad Software, La Jolla CA, USA).

The continuous variables (e.g., patient age, body weight, scan time) are described as average \pm standard deviation (SD) and ranges, and the categorical variables (e.g., patient sex, history of allergies) are presented as counts and percentages. Each characteristic was compared between EOB group and EGCM group using the unpaired Student's $t$ test for numeric data and the Chi-square test or Fisher's exact test (if the expectation of the event was $<5$ ) for categorical data.

The agreement between the bellows grade in two readers was analyzed using Cohen's $\kappa$ for each phase. The agreement between the artifact score in two readers was assessed using Fleiss' $\kappa$ for each phase. Agreement was classified as

1 , which indicates no image deterioration. In contrast, arterial phase image (b) showed artifact score 5, which indicates extensive respiratory motion artifacts with severe image deterioration and images were not acceptable for diagnostic use 
poor (0-0.09), slight (0.1-0.19), fair (0.2-0.39), moderate (0.40-0.59), substantial (0.6-0.79), or excellent (0.8-1.0).

The Jonckheere-Terpstra trend test was used to evaluate the relationship between the abdominal movement measured by bellows grade and the degree of image deterioration shown by artifact score.

Chi-square test and Fisher's exact test were performed for the analysis of incidence and degree of each abdominal movement pattern in EOB- and EGCM-enhanced MRI.

For the analysis of the relationship between the degree of TAM and the frequency of transient artifact in the arterial phase, Cochran-Armitage trend test was applied to the EOB group, and Fisher's exact test was applied to the EGCM group due to no occurrence of severe TAM.

Friedman test was performed for the comparison of the average artifact score on each phase of dynamic contrastenhanced MRI in NAM, mild-TAM and severe-TAM cases. For post hoc analysis, Dunn's test was used for multiple comparisons.

For all tests, $p$ values less than 0.05 were accepted as statistically significant.

\section{Results}

\section{Background characteristics and agreement in assessment}

The patient characteristics of this study are shown in Table 2. There was no statistically significant difference in the patient characteristics and other risk factors between EOB group and EGCM group with exception of hepatitis virus carrier $[65.0 \%$ (134 of 206) vs $3.4 \%$ (4 of 119), $p<0.001$, respectively].

In terms of inter-observer agreement, the Cohen's $\kappa$ coefficients for the bellows grade were 0.79 in precontrast, 0.82 in arterial phase, and 0.83 in portal venous phase, indicating substantial to excellent agreement. The Fleiss' $\kappa$ coefficients for the artifact score were 0.54 in precontrast, 0.67 in arterial phase, and 0.58 in portal venous phase, indicating moderate to substantial agreement.

\section{Relationship between respiratory bellows grade and artifact score: EOB vs EGCM (Table 3)}

In the acquisitions with bellows grade 3, all images showed artifact score of $>2$, while no images had artifact score of $>4$ in the acquisitions with bellows grade 1 in EOB group and artifact score of $>2$ in the acquisitions with bellows grade 1 in EGCM group. The higher average artifact score was associated with the higher bellows grade significantly both in EOB group $(p<0.001)$ and in EGCM group $(p<0.05)$ in any contrast-enhanced phase of dynamic MRI.

\section{Relationship between "transient abdominal movement" and "transient artifact in arterial phase": EOB vs EGCM}

Incidence and degree of TAM in EOB- and EGCMenhanced MRI were as follows: within 206 cases of EOB group, 120 cases showed NAM (58.3\%), 27 cases showed mild-TAM (13.1\%), 8 cases showed severe-TAM $(3.9 \%)$ and 21 cases showed IAM (10.2\%) in EOB group (Table 4). Within 119 cases of EGCM group, 91 cases showed NAM (76.5\%), 4 cases showed mild-TAM (3.4\%), no case showed severe-TAM and 6 cases showed IAM (5.0\%) in EGCM group (Table 4).

There were significant differences in the frequency of both mild and severe TAM between EOB and EGCM groups ( $p=0.003$ and 0.03 , respectively).

Relationship between the abdominal movement pattern and the occurrence of transient artifact in arterial phase in EOB- and EGCM group is shown in Table 5. Both in EOB and EGCM groups, significant positive correlation was shown between the degree of "transient abdominal movement" and the occurrence of "transient artifact in arterial phase" (Table 5). Also, no "transient artifact in arterial phase" was observed in NAM cases in either EOB or EGCM group (Table 5).

The results of comparison of the average artifact score on each phase of dynamic contrast-enhanced MRI in NAM, mild-TAM and severe-TAM cases are shown in Fig. 4. In NAM cases, artifact scores of each contrast phase of the EOB group were as follows: $1.10 \pm 0.29$ on pre-contrast, $1.26 \pm 0.57$ on arterial phase and $1.08 \pm 0.28$ on portal venous phase. And artifact scores of each contrast phase of EGCM group were as follows: $1.11 \pm 0.28$ on pre-contrast, $1.16 \pm 0.32$ on arterial phase and $1.12 \pm 0.29$ on portal phase.

In mild-TAM cases, artifact scores of each contrast phases of EOB group were as follows: $1.16 \pm 0.48$ on pre-contrast, $2.80 \pm 1.06$ on arterial phase and $1.23 \pm 0.39$ on portal venous phase, and artifact score of arterial phase was significantly high compared to that of precontrast and portal phase images $(p<0.0 .001)$. Artifact scores of each contrast phase of EGCM group were as follows: $1.00 \pm 0$ on pre-contrast, $2.08 \pm 1.29$ on arterial phase and $1.17 \pm 0.19$ on portal phase, and artifact score of arterial phase images was significantly high compared to that of precontrast phase $(p<0.05)$, and it was high compared to that of portal phase images but there was no significant difference $(p=0.23)$. 
NAM cases
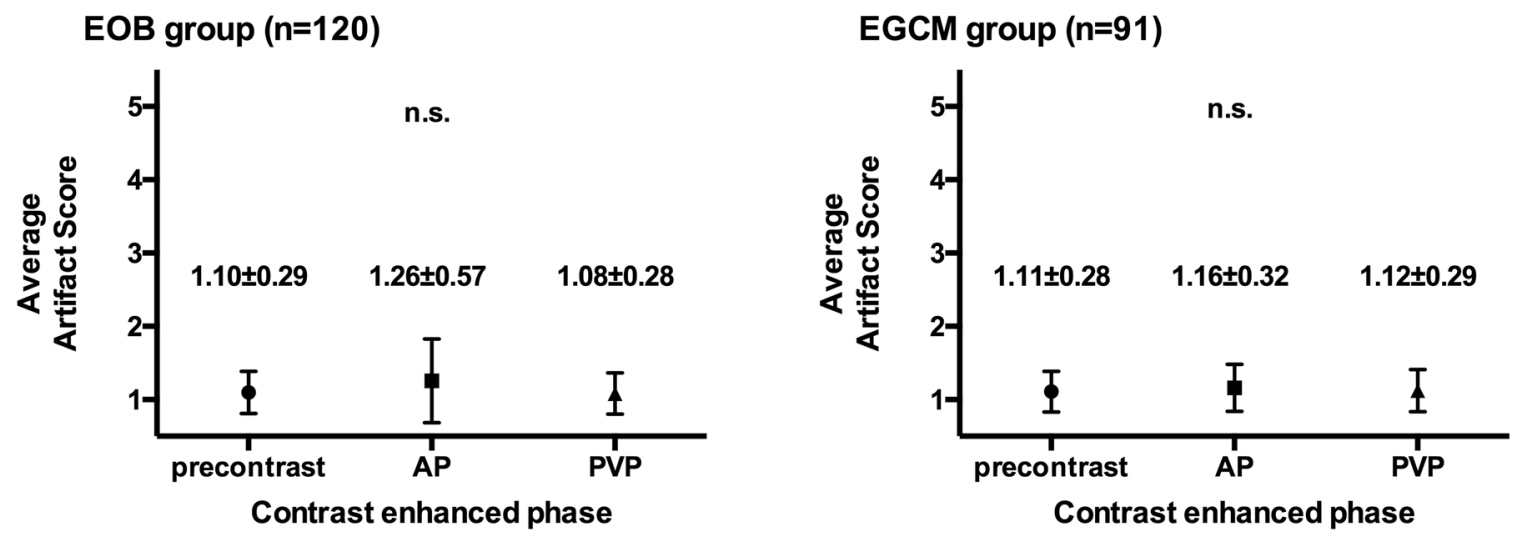

Mild-TAM cases
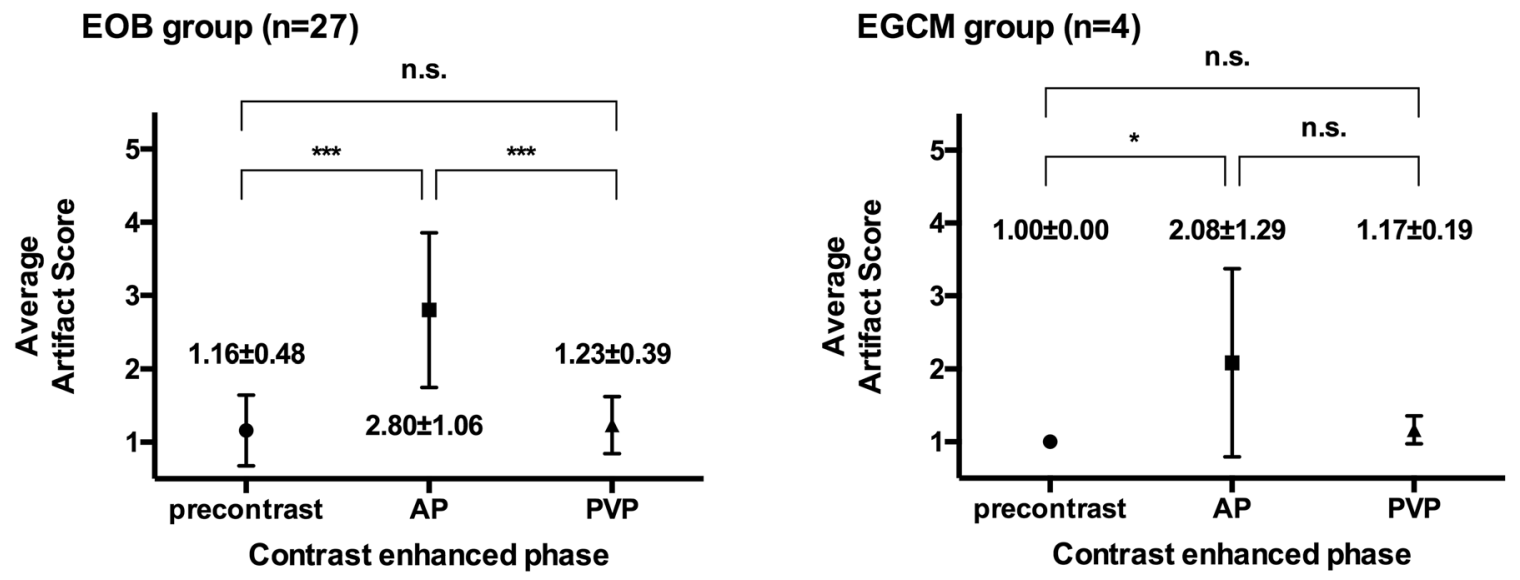

Severe-TAM cases

EOB group $(n=8)$

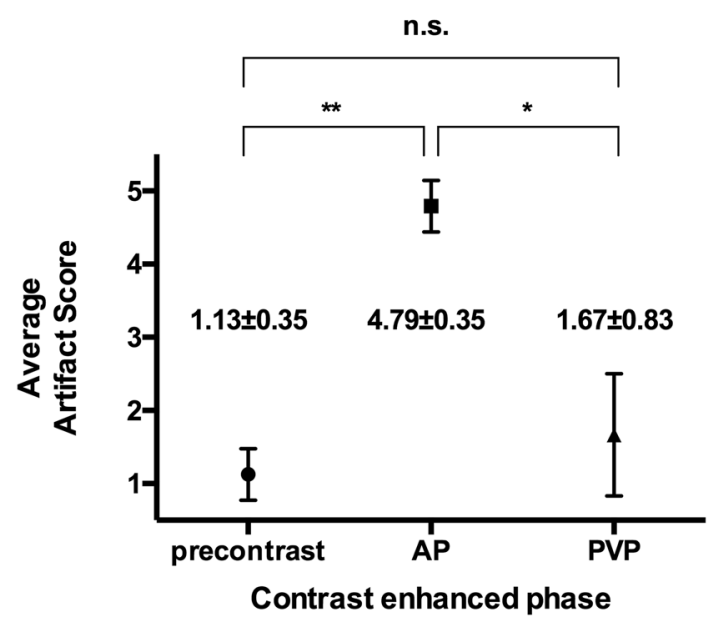


4Fig. 4 Comparison of the average artifact score on each phase of dynamic contrast-enhanced MRI in EOB and EGCM groups of each abdominal movement pattern. NAM no abdominal movement, $T A M$ transient abdominal movement, n.s. not significant. ${ }^{*} p<.05$, $* * p<.01 ; * * * p<.0001$. Friedman test was performed for the comparison of the artifact score on each phase of dynamic contrastenhanced MRI in NAM, mild-TAM and severe-TAM cases. For post hoc analysis, Dunn's test was performed for multiple comparisons

In severe-TAM cases, artifact scores of each contrast phase of EOB group were as follows: $1.13 \pm 0.35$ on precontrast, $4.79 \pm 0.35$ on arterial phase and $1.67 \pm 0.83$ on portal venous phase, and artifact score of arterial phase images showed significantly high compared to that of precontrast $(p<0.01)$ and portal phase images $(p<0.05)$. No cases showed severe TAM in EGCM group.

Although looking at the individual case results of the transient artifact in arterial phase, 4 patients with mild-TAM in EOB group showed transient artifact in arterial phase, and 1 patient with mild TAM in EGCM group showed transient artifact in arterial phase (Table 5); in this average artifact score analysis (Fig. 4), only severe-TAM cases of EOB group (average artifact score $4.79 \pm 0.35$ ) meet our definition of transient artifact in arterial phase (artifact score 4 or 5). And mild-TAM cases of both the contrast material groups did not meet the definition of transient artifact in arterial phase $(2.80 \pm 1.06$ in EOB group and 2.08 \pm 1.29 in EGCM group). Furthermore, NAM cases of both the contrast material groups did not show any artifact in arterial phase $(1.26 \pm 0.57$ in EOB group and $1.16 \pm 0.32$ in EGCM group).

\section{Discussion}

Motion-related artifact is considered to be one of the causes of transient artifact in arterial phase (TSM). However, few studies have paid attention to the relationship between the degree of the abdominal movement during arterial phase image acquisition period and the degree of image deterioration [8-10]. In this study, we tried to show the direct relationship between abdominal movement and artifact.

The respiratory bellows is the device that is used during MR image acquisition, for monitoring abdominal movement caused by respiration, to reduce respiratory motion artifact on MR images [11].

We conducted the semi-quantitative analysis of abdominal movement with the electrogram of the respiratory bellows. Although there are several studies that utilize the respiratory bellows for monitoring abdominal movement qualitatively $[8,10,12]$, as far as we know, our study is the first report that utilizes the electrogram of the respiratory bellows for semi-quantitative analysis of abdominal movement during MR image acquisition period.
According to our results in Table 3, the higher the bellows grade, the higher the significance of average artifact score, both in the EOB group and in EGCM group. It means the degree of abdominal movement intimately correlated with the image quality in any contrast phase irrespective of the contrast agent type.

Davenport et al. [13] speculated that TSM of contrastenhanced MRI originates from drug-induced respiratory movement. According to their theory, abdominal movement might be observed only on arterial phase of contrast-enhanced MRI and no abdominal movement might be observed both on precontrast and portal venous phase images.

Therefore, we defined this abdominal movement pattern as TAM pattern and examined the incidence and degree of TAM in EOB- and EGCM-enhanced MR studies.

Interestingly, between EOB and EGCM groups, the incidence of NAM and TAM showed significant difference, namely frequency of TAM is significantly high in EOB compared to EGCM as shown in Table 4. This result is possibly caused by the difference in the pharmacological effect of EOB and EGCM. Further animal experimental study might be necessary to clarify this issue.

In our result, $17 \%$ of the patients showed TAM in EOB group. In detail, $13.1 \%$ showed mild-TAM, and $3.9 \%$ showed severe-TAM in EOB group. In EGCM group, 3.4\% of the patients showed mild-TAM, and no cases showed severe-TAM.

Based on the previous reports, the incidence of transient artifact in arterial phase in EOB- and in EGCM-enhanced MR examination are $18 \%$ and $2 \%$ in Davenport et al. [5], $10.7 \%$ and $0.5 \%$ in Pietryga et al. [6], and $21.5 \%$ and $3.1 \%$ in Motosugi et al. [8].

The incidence of TAM in our result is similar to the frequency of the previously reported transient artifact in arterial phase. From this similarity, we can speculate that TAM might be closely related to the occurrence of transient artifact in arterial phase.

In terms of the relationship between the abdominal movement pattern and the occurrence of transient artifact in arterial phase in EOB and EGCM groups shown in Table 5, significant positive correlation was shown between the degree of "transient abdominal movement" and the occurrence of "transient artifact in arterial phase". This result supported our hypothesis that abdominal movement is necessary for the occurrence of transient artifact in arterial phase.

As well, since a considerable number of mild-TAM cases did not show transient artifact in arterial phase, we could surmise that the degree of these mild TAMs did not reach the threshold for the occurrence of transient artifact in the arterial phase.

From our comparison analysis of the average artifact score on each phase of dynamic contrast-enhanced MRI 
Table 2 Patient characteristics of this study

\begin{tabular}{|c|c|c|c|}
\hline & Gd-EOB-DTPA & EGCM & $p$ value \\
\hline No. of patients & 206 & 119 & \\
\hline Mean age $(\text { years })^{\mathrm{a}}$ & $65.5 \pm 9.6(33-83)$ & $66.1 \pm 12.0(35-78)$ & 0.63 \\
\hline No. of woman & 78 (37.9) & $57(47.9)$ & 0.11 \\
\hline Mean patient weight $(\mathrm{kg})^{\mathrm{a}}$ & $59.5 \pm 11.3(34.4-99.0)$ & $58.8 \pm 10.0(48.0-81.2)$ & 0.58 \\
\hline Mean body mass index $\left(\mathrm{kg} / \mathrm{m}^{2}\right)^{\mathrm{a}}$ & $23.3 \pm 3.9(16.5-40.4)$ & $23.0 \pm 2.9(17.9-29.4)$ & 0.28 \\
\hline Smoking exposure (pack-years) ${ }^{\mathrm{a}}$ & $22.3 \pm 29.6(0-150)$ & $18.9 \pm 29.7(0-150)$ & 0.31 \\
\hline Whole allergies & $71(34.5)$ & $41(34.5)$ & 0.99 \\
\hline Asthma & $2(1.0)$ & $4(3.4)$ & 0.20 \\
\hline Allergy to iodine-containing media & $20(10.2)$ & $6(5.0)$ & 0.14 \\
\hline Allergy to gadolinium-based contrast media & $0(0.0)$ & $0(0.0)$ & NA \\
\hline Hepatitis virus & $134(65.0)$ & $4(3.4)$ & $<0.001$ \\
\hline HBV carrier & $33(16.0)$ & $2(1.7)$ & $<0.001$ \\
\hline HCV carrier & $101(49.0)$ & $2(1.7)$ & $<0.001$ \\
\hline \multicolumn{4}{|l|}{ Pleural effusion } \\
\hline Mild & $4(1.5)$ & $1(0.8)$ & 0.66 \\
\hline Moderate to large & $0(0.0)$ & $1(0.8)$ & 0.37 \\
\hline \multicolumn{4}{|l|}{ Ascites } \\
\hline Mild & $2(1.0)$ & $0(0.0)$ & 0.53 \\
\hline Moderate to large & $0(0.0)$ & $0(0.0)$ & NA \\
\hline Scan time $(s)^{\mathrm{a}}$ & $15.0 \pm 2.0(12-20)$ & $14.9 \pm 1.60(12-19)$ & 0.70 \\
\hline Arterial phase timing after the injection $(\mathrm{s})^{\mathrm{a}}$ & $24.9 \pm 3.2(19-40)$ & $25.0[$ fixed $]$ & 0.81 \\
\hline
\end{tabular}

Except where indicated, data are numbers of patients, with percentages in parentheses

$N A$ not applicable

* $P$ values were calculated with the Student's $t$, the Chi-square, or Fisher's exact test, as appropriate

${ }^{a}$ Data are means \pm standard deviations. Number in parentheses is the ranges in EOB and EGCM groups of each abdominal movement pattern, in NAM cases, the average artifact score ranged within 1.08-1.26 in all phases of both in EOB and EGCM groups. It means there is no image deterioration irrespective of the contrast agent type in no abdominal movement cases. If the occurrence of transient artifact in the arterial phase and the abdominal movement are unrelated, artifact score on arterial phase may show to be significantly higher than other phases even in NAM cases, but there was no significant difference in artifact score among the phases. This result also supported our hypothesis that abdominal movement is the direct cause of artifact.

In mild-TAM cases, the average artifact score was 1.16 on precontrast, 2.80 on arterial, and 1.23 on portal venous phase in EOB group, and the average artifact score was 1.00 on precontrast, 2.08 on arterial, and 1.16 on portal venous phase in EGCM group. It means, although there were no image deteriorations on both precontrast and portal venous phase, image deterioration is observed on arterial phase images irrespective of the contrast agent type in mild-TAM cases.

However, since the average artifact scores of the arterial phases both in EOB group and in EGCM group did not reach the threshold value for our definition of transient artifact in arterial phase, mild-TAM cases were not regarded to be positive transient artifact in the arterial phase.

In severe-TAM cases, the average artifact score was 1.13 on precontrast, 4.79 on arterial, and 1.67 on portal venous phase in EOB group. It means, although there were no image deteriorations on both precontrast and portal venous phases, severe image deterioration is observed on arterial phase images in EOB group in severe-TAM cases. Since the average artifact scores of the arterial phases in EOB group reach the threshold value for our definition of transient artifact in the arterial phase, severe-TAM cases were considered to be positive transient artifact in the arterial phase.

Based on these results, we could confirm that transient artifact in the arterial phase is intimately associated with abdominal movement which occurs only during the arterial phase image acquisition period of contrast-enhanced MRI. In addition, we have elucidated that among positive TAM cases during the arterial phase, only severe abdominal movement cases are recognized as transient artifact in the arterial phase.

Our study has several limitations. First, in terms of the patient characteristics and other risk factors, there was a significant difference between EOB group and EGCM group in the incidence of positive hepatitis virus cases. Since 
Table 3 Relationship between respiratory bellows grade and average artifact score

\begin{tabular}{lll}
\hline & \multicolumn{3}{l}{ Average artifact score } \\
\cline { 2 - 4 } & $2-4$ & $2-4$ \\
\hline
\end{tabular}

(a) Bellows grade

EOB

$\begin{array}{crrr}3 & 0 & 6 & 19 \\ 2 & 61 & 49 & 11 \\ 1 & 450 & 22 & 0 \\ \text { EGCM } & & & \\ 3 & 0 & 2 & 9 \\ 2 & 46 & 4 & 0 \\ 1 & 296 & 0 & 0\end{array}$

(b) Bellows grade

EOB

$\begin{array}{crrrr}3 & 0 & 2 & 1 & p<0.0001 \text { for trend } \\ 2 & 17 & 10 & 0 & \\ 1 & 170 & 6 & 0 & \\ \text { EGCM } & & & & \\ 3 & 0 & 1 & 2 & p<0.048 \text { for trend } \\ 2 & 14 & 1 & 0 & \\ 1 & 101 & 0 & 0 & \end{array}$

(c) Bellows grade

EOB

$\begin{array}{crrrr}3 & 0 & 1 & 14 & p<0.0001 \text { for trend } \\ 2 & 20 & 31 & 8 & \\ 1 & 121 & 11 & 0 & \\ \text { EGCM } & & & & p<0.017 \text { for trend } \\ 3 & 0 & 0 & 4 & \\ 2 & 19 & 2 & 0 & \\ 1 & 94 & 0 & 0 & \end{array}$

(d) Bellows grade

$\begin{array}{crrrr}\text { EOB } & & & & \\ 3 & 0 & 3 & 4 & p<0.0001 \text { for trend } \\ 2 & 24 & 8 & 3 & \\ 1 & 159 & 5 & 0 & \\ \text { EGCM } & & & & \\ 3 & 0 & 1 & 3 & p<0.019 \text { for trend } \\ 2 & 13 & 1 & 0 & \\ 1 & 101 & 0 & 0 & \end{array}$

(a) Overall images, (b) precontrast ***images, (c) arterial phase images, (d) portal venous phase images

${ }^{*} p$ values were calculated with the Jonckheere-Terpstra trend test

EOB-enhanced MR study is mainly conducted for detection and characterization of hepatic tumor in high-risk patients for hepatocellular carcinoma, it is reasonable that the proportion of positive hepatitis virus cases in EOB group is bigger than EGCM group, which is mainly conducted for closer examination of other upper abdominal malignancies such as pancreatic cancer, gallbladder cancer and so on.

Second, the incidence of transient artifact in the arterial phase in EOB group is higher than that in EGCM group, but this difference was not significant. The possible reasons for these differences may be attributed to the following two reasons. The first one is the dose of EOB; it was relatively low when compared with previous studies because we determined the amount of contrast media by the patient body weight. Davenport et al. [13] reported that the volume of injected contrast media affected TSM significantly, and our findings supported their data. The second one is the injection speed of EOB; we administrated EOB at a speed of $1.0 \mathrm{~mL} / \mathrm{s}$. Tanimoto et al. [14] reported slower injection rate could reduce artifacts in gadoxetic acid-enhanced dynamic MR imaging $(1.5 \mathrm{~mL} / \mathrm{s}$ vs $3.0 \mathrm{~mL} / \mathrm{s}$ ).

Third, we have excluded IAM pattern $(n=21$ in EOB group and $n=6$ in EGCM group) that do not fulfill NAM or TAM criteria in comparison of the average artifact score on each phase of dynamic contrast-enhanced MRI in EOB and EGCM groups of each abdominal movement pattern. The reason is as follows. Some of IAM cases were no abdominal movement both on precontrast and arterial phase, and showed abdominal movement on portal venous phase. This type of IAM is considered to be a delayed-type TAM, and it is possible that the abdominal movement caused by the pharmacological action of the contrast agent may have occurred somewhat later than the typical timing. And the other IAM cases were no abdominal movement only on precontrast phase, and both arterial and portal venous phase showed various degrees of abdominal movement. This type of IAM is suspected to be an extended type TAM due to delayed elimination of the pharmacological effect of the contrast agent compared with typical timing. However, since there are no effective methods to confirm these speculations and these atypical cases are small in number, we have excluded these cases as atypical respiratory patterns. Animal experimental study might be effective to clarify such pharmacological effect issue of contrast material.

Fourth, since this study is retrospective analysis, EGCM group contains several kinds of different contrast agents.

In conclusion, irrespective of contrast material types, image quality is highly correlated with abdominal movement measured by respiratory bellows. Moreover, artifact in arterial phase was not observed in NAM, which indicated that the abdominal movement is the direct cause of artifact. 
Table 4 Incidence of each abdominal movement pattern in EOB- and EGCM-enhanced MRI

\begin{tabular}{|c|c|c|c|c|c|c|c|c|}
\hline & NAM & & Mild-TAM & & Severe-TA & & IAM & $\begin{array}{c}\text { Difficulty } \\
\text { in breath hold }\end{array}$ \\
\hline $\begin{array}{c}\text { EOB } \\
(n=206)\end{array}$ & $\begin{array}{c}120 \\
(58.3 \%)\end{array}$ & $*$ & $\left.\begin{array}{c}27 \\
(13.1 \%)\end{array}\right]$ & ** & $\begin{array}{c}8 \\
(3.9 \%)\end{array}$ & $* * *$ & $\begin{array}{c}21 \\
(10.2 \%)\end{array}$ & $\begin{array}{c}30 \\
(14.5 \%)\end{array}$ \\
\hline $\begin{array}{l}\text { EGCM } \\
(n=119)\end{array}$ & $\begin{array}{c}91 \\
(76.5 \%)\end{array}$ & & $\begin{array}{c}4 \\
(3.4 \%)\end{array}$ & & $\begin{array}{c}0 \\
(0 \%)\end{array}$ & & $\begin{array}{c}6 \\
(5.0 \%)\end{array}$ & $\begin{array}{c}18 \\
(15.1 \%)\end{array}$ \\
\hline
\end{tabular}

$N A M$ no-abdominal movement, TAM transient abdominal movement, IAM irregular abdominal movement, $* p<0.003, * * p=0.003, * * * p=0.03$. $p$ values were calculated with the Chi-square test or Fisher's exact test (if the expectation of the event was $<5$ )

Table 5 Relationship between the abdominal movement pattern and the occurrence of transient artifact in the arterial phase in EOB and EGCM groups

Transient artifact in arterial phase

\begin{tabular}{ccccc} 
& & negative & positive & \\
\hline \multirow{3}{*}{ EOB } & Severe-TAM & 1 & 7 & \\
& Mild-TAM & 23 & 4 & $\begin{array}{l}\text { P<0.001 } \\
\text { for trend }\end{array}$ \\
& NAM & 120 & 0 & \\
\hline \multirow{2}{*}{ EGCM } & Severe-TAM & 0 & 0 & \\
& Mild-TAM & 3 & 1 & \\
& NAM & 91 & 0 & ]$P=0.04$ \\
\hline
\end{tabular}

TAM transient abdominal movement, NAM no-abdominal movement, $p$ values were calculated with Cochran-Armitage trend test in EOB group. In EGCM group, that was calculated by Fisher's exact test due to no occurrence of severe TAM

Acknowledgements This research was supported by JSPS KAKENHI Grant number JP18K07669.

\section{Compliance with ethical standards}

Conflict of interest All the authors and our institution have no conflict of interest.

Ethical approval This manuscript has not been published and is not under consideration for publication elsewhere. All the authors have read the manuscript and have approved this submission (Responsible authority of our Departments, Toshifumi Gabata, is one of the authors and explicitly approved this submission).

\section{References}

1. Frydrychowicz A, Lubner MG, Brown JJ, Merkle EM, Nagle SK, Rofsky NM, et al. Hepatobiliary MR imaging with gadolinium-based contrast agents. J Magn Reson Imaging. 2012;35(3):492-511.

2. Sirlin CB, Hussain HK, Jonas E, Kanematsu M, Min Lee J, Merkle EM, et al. Consensus report from the 6th international forum for liver MRI using gadoxetic acid. J Magn Reson Imaging. 2014;40(3):516-29.

3. Merkle EM, Zech CJ, Bartolozzi C, Bashir MR, Ba-Ssalamah A, Huppertz A, et al. Consensus report from the 7th international forum for liver magnetic resonance imaging. Eur Radiol. 2016;26(3):674-82.

4. Davenport MS, Viglianti BL, Al-Hawary MM, Caoili EM, Kaza RK, Liu PS, et al. Comparison of acute transient dyspnea after intravenous administration of gadoxetate disodium and gadobenate dimeglumine: effect on arterial phase image quality. Radiology. 2013;266(2):452-61.

5. Davenport MS, Caoili EM, Kaza RK, Hussain HK. Matched within-patient cohort study of transient arterial phase respiratory motion-related artifact in MR imaging of the liver: gadoxetate disodium versus gadobenate dimeglumine. Radiology. 2014;272(1):123-31.

6. Pietryga JA, Burke LM, Marin D, Jaffe TA, Bashir MR. Respiratory motion artifact affecting hepatic arterial phase imaging with gadoxetate disodium: examination recovery with a multiple arterial phase acquisition. Radiology. 2014;271(2):426-34.

7. Bashir MR, Castelli P, Davenport MS, Larson D, Marin $\mathrm{D}$, Hussain HK, et al. Respiratory motion artifact affecting hepatic arterial phase MR imaging with gadoxetate disodium is more common in patients with a prior episode of arterial phase motion associated with gadoxetate disodium. Radiology. 2015;274(1):141-8. 
8. Motosugi U, Bannas P, Bookwalter CA, Sano K, Reeder SB. An investigation of transient severe motion related to gadoxetic acidenhanced MR imaging. Radiology. 2016;279(1):93-102.

9. Davenport MS, Malyarenko DI, Pang Y, Hussain HK, Chenevert TL. Effect of gadoxetate disodium on arterial phase respiratory waveforms using a quantitative fast Fourier transformation-based analysis. Am J Roentgenol. 2017;208(2):328-36.

10. Park YS, Lee CH, Yoo JL, Kim IS, Kiefer B, Woo ST, et al. Hepatic arterial phase in gadoxetic acid-enhanced liver magnetic resonance imaging: analysis of respiratory patterns and their effect on image quality. Investig Radiol. 2016;51(2):127-33.

11. Ehman RL, McNamara MT, Pallack M, Hricak H, Higgins CB. Magnetic resonance imaging with respiratory gating: techniques and advantages. Am J Roentgenol. 1984;143(6):1175-82.

12. Kromrey ML, Hori M, Goshima S, Kozaka K, Hyodo T, Nakamura $\mathrm{Y}$, et al. Gadoxetate disodium-related event during image acquisition: a prospective multi-institutional study for better MR practice. Eur Radiol. 2019. https://doi.org/10.1007/s00330-01906358-7(Epub ahead of print).

13. Davenport MS, Bashir MR, Pietryga JA, Wever JT, Khalatbari S, Hussain HK. Dose-toxicity relationship of gadoxetate disodium and transient severe respiratory motion artifact. Am J Roentgenol. 2014;203(4):796-802.

14. Tanimoto A, Higuchi N, Ueno A. Reduction of ringing artifacts in the arterial phase of gadoxetic acid-enhanced dynamic MR imaging. Magn Reson Med Sci. 2012;11(2):91-7.

Publisher's Note Springer Nature remains neutral with regard to jurisdictional claims in published maps and institutional affiliations. 